\title{
Nouvelle mention, pour le «Midi》 méditerranéen, de populations naturelles anautogènes et sténogames de Culex pipiens pipiens L.
}

par N. PASTeUR, J.A. RIOUX, E. GUILVARD et J. PECH-PERIERES

(Collaboration technique: A. BELmonte)

Laboratoire d'Ecologie médicale et Pathologie parasitaire ( $\mathrm{P}^{\mathrm{r}} \mathrm{J}$.-A. Rioux),

Faculté de Médecine, F 34000 Montpellier

\section{Résumé.}

Les auteurs mentionnent l'existence, en Languedoc méditerranéen, d'une population anautogène et sténogame de Culex pipiens. L'anautogénèse a été confirmée jusqu’à la $10^{\epsilon}$ génération. Le morphogramme de Callot correspond à la forme anautogène. A cette occasion se repose le problème du type de liaison, génétique ou écologique, des caractères classiquement associés à l'autogénèse.

\section{Summary.}

A new report of naturally anautogenous and stenogamic populations of Culex pipiens pipiens $L$. in the south of France.

The presence of an anautogenous and stenogamic population of Culex pipiens pipiens in Languedoc, France, is reported. The anautogeny was confirmed through 10 generations. A Callot morphogram corresponded to the anautogenous form. These results raise again the question of the type of relationships, genetic or ecological, of the characters classically associated with autogeny.

Accepté le 2 octobre 1976. 
Depuis la découverte des variations électrophorétiques des systèmes enzymatiques, les recherches génétiques sur le complexe Culex pipiens ont pris un nouvel essor. Les facilités de manipulation de ces systèmes, leur simplicité, leurs relations de plus en plus évidentes avec les facteurs mésologiques, en font un remarquable outil d'analyse.

Ce regain d'intérêt conduit actuellement à tenter l'isolement de nouvelles souches, de manière à reprendre l'étude des « races » autogènes et anautogènes, antérieurement définies par E. Roubaud (tableau I).

TABLEAU I. - Caractéristiques éthologiques des « formes » autogène et anautogène.

\begin{tabular}{|c|c|}
\hline « Forme » autogène & *Forme » anautogène \\
\cline { 3 - 3 } Autogène & Anautogène \\
Sténogame & Eurygame \\
Anthropophile & Zoophile \\
Homodyname & Hétérodyname \\
Hypogée & Epigée \\
\hline
\end{tabular}

C'est à l'occasion d'une telle étude, menée en Languedoc-Roussillon, que nous avons individualisé une population possédant des caractéristiques comportementales intermédiaires. Nous en présentons succinctement l'observation.

Le 16 septembre 1974, nous effectuons un prélèvement de larves de Culex pipiens dans un abreuvoir traditionnel ( lavogne») situé en garrigue, le long de la route $\mathrm{D} 122$, près du lieu-dit Frouzet, à $4 \mathrm{~km}$ du Causse-de-la-Selle (Hérault). Les larves transportées au laboratoire, sont nourries de biscuits de chiens. Les adultes sont placés en cages $40 \times 40 \times 40 \mathrm{~cm}$. La sténogamie, appréciée d'après la fertilité des pontes, varie entre $20 \%$ et $80 \%$ (tableau II).

L'autogénèse est éprouvée jusqu'à la $10^{\circ}$ génération, selon la technique antérieurement définie (dissection du $20^{\circ}$ jour) : les follicules ovariens de la génération parentale $\mathrm{F}_{0}$ et pour une part de la $\mathrm{F}_{1}$ à la $\mathrm{F}_{4}$, restent bloqués au stade $\mathrm{I}$ de Christophers. A partir de la $F_{5}$, la totalité atteint le stade II sans toutefois le dépasser (tableau III). En d'autres termes, la population observée reste anautogène pendant 'toute la durée de l'étude. Malgré un indice siphonique bas (1), le morphogramme de Callot, établi à la $\mathrm{F}_{10}$, montre une disposition caractéristique de la « forme » anautogène de $C$. pipiens pipiens (fig. 1). L'étude du comportement de piqûre indique une nette préférence pour le Pigeon (tableau IV).

(1) L'indice siphonique $\left(\frac{\mathrm{L}}{1}\right)$ est plus élevé chez les mâles (fig. 2). 


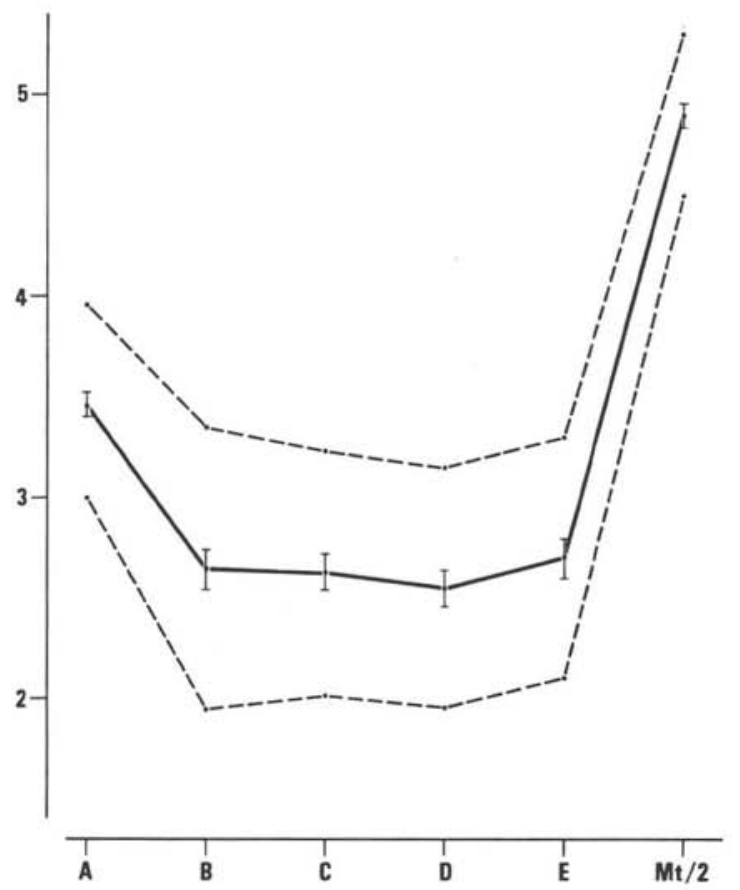

FIG. 1. - Culex pipiens pipiens, souche Frouzet: morphogramme de Callot, établi sur l'ensemble de cinq pontes $\left(\mathrm{F}_{10}\right)$. (Les traits interrompus limitent les écarts-types des échantillons, le trait continu correspond aux moyennes, les barres verticales représentent les intervalles de confiance des moyennes).

Tableau II. - Fertilité estimée sur les pontes

\begin{tabular}{|c|c|c|c|}
\hline \multirow{2}{*}{ Générations } & \multirow{2}{*}{ Pontes totales } & \multicolumn{2}{|c|}{ Pontes fertiles } \\
\hline & & $\mathrm{n}^{\circ}$ & $\%$ \\
\hline $\mathrm{F}_{1}$ & 87 & 19 & 21,84 \\
\hline $\mathrm{F}_{2}$ & 13 & 8 & 61,54 \\
\hline $\mathrm{F}_{3}$ & 76 & 22 & 28,95 \\
\hline $\mathrm{F}_{4}$ & 55 & 25 & 45,45 \\
\hline $\mathrm{F}_{5}$ & 12 & 10 & 83,33 \\
\hline $\mathrm{F}_{0}$ & 120 & 57 & 47,50 \\
\hline
\end{tabular}



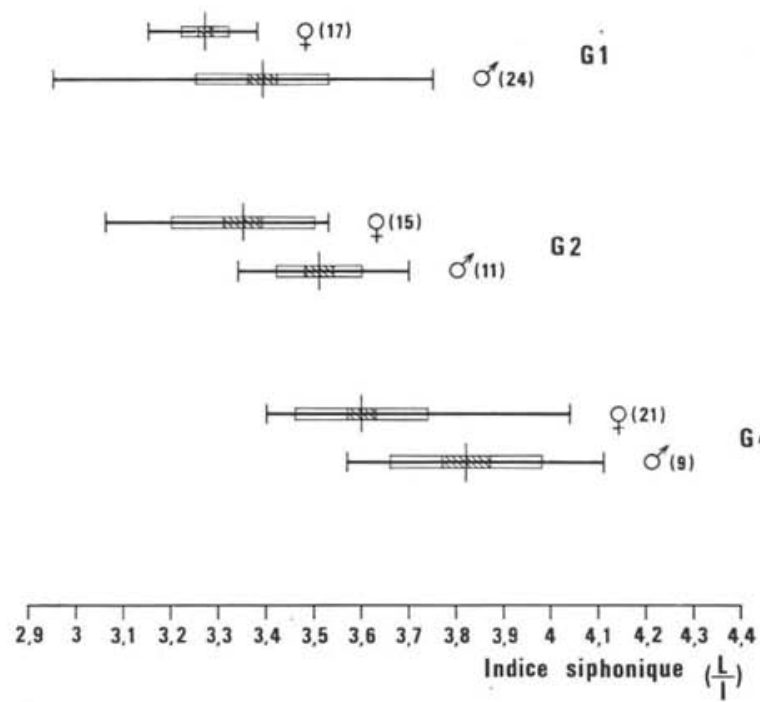

FIG. 2. - Culex pipiens pipiens, souche Frouzet: indices siphoniques des individus $\delta$ et $q$ calculés sur trois pontes $\left(\mathrm{F}_{10}\right)$. Dans les trois cas, les siphons des larves $\delta$ sont plus longs que ceux des larves $\uparrow(P<0,05)$. (Trait vertical : moyenne de l'échantillon; rectangle hachuré : intervalle de confiance de la moyenne; rectangle blanc: écart-type de l'échantillon; ligne horizontale : extrêmes observés).

TABLEAU III. - Vitellogénèse appréciée selon l'échelle de Christophers. Noter l'arrêt du développement au stade II.

\begin{tabular}{|c|c|c|c|c|c|c|}
\hline \multirow{2}{*}{ Générations } & \multirow{2}{*}{$\mathrm{n}^{\circ} q$} & \multicolumn{5}{|c|}{ Stades ovariens (Christophers) } \\
\hline & & I & II & III & IV & V \\
\hline $\mathrm{F}_{0}$ & 194 & 190 & 4 & & 0 & \\
\hline$F_{1}$ & 1137 & 222 & 915 & & 0 & \\
\hline $\mathrm{F}_{2}$ & 343 & 29 & 314 & & 0 & \\
\hline $\mathrm{F}_{3}$ & 337 & 10 & 327 & & 0 & \\
\hline $\mathrm{F}_{4}$ & 170 & 4 & 166 & & 0 & \\
\hline $\mathrm{F}_{5}$ & 111 & 0 & 111 & & 0 & \\
\hline $\mathrm{F}_{6}$ & 96 & 0 & 96. & & 0 & \\
\hline $\mathrm{F}_{\overline{7}}$ & 81 & 0 & 81 & & 0 & \\
\hline $\mathrm{F}_{8}$ & 93 & 0 & 93 & & 0 & \\
\hline$F_{9}$ & 103 & 0 & 103 & & 0 & \\
\hline$F_{10}$ & 80 & 3 & 77 & & 0 & \\
\hline
\end{tabular}


TABLEAU IV. - Préférences trophiques Mammifère/Oiseau

\begin{tabular}{|c|c|c|}
\hline Hôte & $\mathrm{n}^{\circ} \uparrow$ \\
\hline Lapin & 90 \\
\hline Pigeon & 90 & $\frac{\mathrm{n}^{\circ}}{4}$ \\
\hline 14 & $\frac{4,44}{15,55}$ \\
\hline
\end{tabular}

Une telle observation confirme l'existence dans le sud de la France de populations de Culex pipiens, à la fois sténogames et anautogènes, se rapprochant par conséquent de la «race berbericus» d'E. Roubaud (1933). Rappelons que cette «race» décrite à tort (2) du Nord de l'Algérie, a été redéfinie comme strictement anautogène, à partir de populations du sud de la France.

Le problème est dès lors reposé d'une éventuelle pléiotropie des gènes autogènes. S'il en était ainsi, ces gènes agiraient à la fois sur le déroulement de la vitellogénèse et le déterminisme des comportements d'accouplement, d'alimentation et de diapause hivernale. Dans le cas inverse, il s'agirait d'une simple liaison écologique, c'est-à-dire dépendant de la concomitance de facteurs mésologiques, tels que la charge nutritive et la température des biotopes aquatiques ou la nature ouverte ou fermée des milieux terrestres. Rappelons qu'en 1960, à la suite de l'analyse d'une population originaire du Tibesti méridional (Faya), nous nous étions orientés vers cette deuxième hypothèse ; les souches observées étant à la fois eurygames et autogènes.

Au demeurant, une telle observation permet dès à présent d'envisager, sans difficulté excessive, l'étude génétique du caractère autogène dans l'espèce Culex pipiens. De fait, les études antérieures ont été réalisées en grande partie à partir des populations eurygames, issues directement du terrain et, par là-même, souvent hétérozygotes pour le caractère autogène.

\section{Bibliographie}

Callot (J.), 1947. - Etude sur quelques souches de Culex pipiens (s. 1.) et sur leurs hybrides. Ann. Parasitol. hum. comp., 22, 380-393.

Callot (J.), 1955. - Etudes sur les hybrides des biotypes de Culex pipiens L. Ann. Parasitol. hum. comp., 30, 363-373.

Callot (J.) et Dao Van Ty, 1943. - Sur quelques souches françaises de Culex pipiens L. Bull. Soc. Path. exot., 36, 229-231.

Lacour (P.), 1937. - Etude biologique sur la race rurale de Culex pipiens L. Thèse médecine, P. Vallier édit., Clermont-Ferrand, 125 p.

PASteur (N.), 1975. - Leucine-aminopeptidases du moustique Culex pipiens: génétique formelle d'un locus chez l'imago. C.R. Acad. Sci., 280, 113-116.

(2) Il s'agissait en réalité de populations présentant, à des taux divers, le caractère autogène. 
Pasteur (N.) et Sinegre (G.), 1975. - Esterase polymorphism and sensitivity to Dursban organophosphorous insecticide in Culex pipiens pipiens populations. Biochem. Genet., 13, 789-803.

Rioux (J.-A.) et Ресн (J.), 1958. - A propos de la biosystématique du «complexe» Culex pipiens. Perspectives nouvelles. C.R. $83^{\circ}$ Congr. Soc. Sav., 597-609.

Rioux (J.-A.), Pech (J.) et Maistre (O.), 1960. - Présence du caractère autogène dans les populations borkouannes de Culex pipiens L. (Diptera-Culicidae). In: Mission épidémiologique au Nord-Tchad, pp. 53-92 P.R.O.H.U.Z.A., édit., Paris.

Roubaud (E.), 1933. - Essai synthétique sur la vie du Moustique commun (Culex pipiens). Ann. Sci. nat. Zool., 16, 163 p.

Roubaud (E.) et Ghelelovitch (S.), 1956. - Observations sur le Moustique anthropophile méditerranéen du groupe pipiens (Culex berbericus). C.R. Acad. Sci., 242, 29002903. 